\title{
Segmentation of 3D EBSD Data for Subgrain Boundary Identification and Feature Characterization
}

\author{
Andrew Loeb ${ }^{1, *}$, Michael Ferry ${ }^{2}$, Lori Bassman ${ }^{1}$ \\ ${ }^{1}$ Department of Engineering, Harvey Mudd College, 301 Platt Blvd, Claremont, CA 91711, USA \\ ${ }^{I}$ School of Materials Science and Engineering, The University of New South Wales, NSW 2052, Australia
}

\section{Abstract}

2 Subgrain structures formed during plastic deformation of metals can be observed by electron

3 backscatter diffraction (EBSD) but are challenging to identify automatically. We have adapted a

4 2D image segmentation technique, fast multiscale clustering (FMC), to 3D EBSD data using a

5 novel variance function to accommodate quaternion data. This adaptation, which has been

6 incorporated into the free open source texture analysis software package MTEX, is capable of

7 segmenting based on subtle and gradual variation as well as on sharp boundaries within the data.

8 FMC has been further modified to group the resulting closed 3D segment boundaries into distinct

9 coherent surfaces based on local normals of a triangulated surface. We demonstrate the excellent

10 capabilities of this technique with application to 3D EBSD data sets generated from cold rolled

11 aluminum containing well-defined microbands, cold rolled and partly recrystallized extra low

12 carbon steel microstructure containing three magnitudes of boundary misorientations, and

13 channel-die plane strain compressed Goss-oriented nickel crystal containing microbands with

14 very subtle changes in orientation.

15

16 Keywords: Electron Backscatter Diffraction, 3D EBSD, Segmentation, Fast Multiscale

17 Clustering, Subgrain Structures, Kuwahara Filter

18

\footnotetext{
* Principal corresponding author.

Tel. +1 360261 3237, E-mail: ael89@ cornell.edu

Present address: 657 Rhodes Hall, Ithaca, NY 14852

(c) 2015. This manuscript version is made available under the Elsevier user license

http://www.elsevier.com/open-access/userlicense/1.0/
} 


\section{1. Introduction}

\section{$20 \quad 1.1 \quad$ Motivation and Background}

21 Studies of the geometric and crystallographic features of microstructures generated by

22 deformation, annealing, and phase transformation can shed light on both their mechanisms of

23 formation, and how they relate to key properties of the materials. A significant characteristic of

24 many types of deformed metals and alloys is the subgrain structure generated. This structure is

25 regularly investigated by electron backscatter diffraction (EBSD) based on local crystallographic

26 orientation measurements. Since regions belonging to a grain share similar orientations, the grain

27 can be reconstructed and then quantitatively characterized from processed EBSD data [1].

28 Furthermore, EBSD techniques can produce three-dimensional (3D) crystallographic data for a

29 sample volume, which is necessary to gain a more complete understanding of the structure than

30 can be taken from two-dimensional (2D) data [2, 3]. From a 3D reconstruction, many geometric

31 and crystallographic features may be measured directly, such as grain volume, shape, and the

32 distribution of misorientations across a boundary [4]. Moreover, a 2D study cannot yield the

33 information necessary for a full crystallographic characterization of grain boundaries or the

34 correlation of a specific geometry with crystallographic orientation. This information is

35 straightforward to extract from 3D EBSD data that has been segmented by grains, and any

36 analog can be similarly found from a segmentation based on subgrain structure.

38 A volumetric EBSD data set that has been segmented into subgrain features can be interrogated

39 for relationships between the detailed microstructure and bulk material properties. However,

40 unlike high angle grain boundaries (HAGBs), the variations between subgrain structures are

41 more subtle in magnitude (low total difference in orientation between subgrains) and spatially 
42 gradual (low point-to-point differences). This makes the identification of subgrain boundaries

43 challenging and, hence, creates a significant obstacle to generating reliable data on the spatial

44 distribution of subgrains within the overall deformation substructure for understanding the

45 mechanisms of subgrain formation.

47 The identification of features in a 3D EBSD data set may be achieved by grouping points that are 48 not separated by a boundary with misorientation higher than a user-defined threshold. This is a 49 common method in commercial software but fails when applied to subgrain data since there is no 50 threshold angle that identifies the boundaries of interest without being dominated by noise in the 51 sample. Figure 1a-c shows an artificially-constructed volume of orientation data representing a 52 gradual transition between two similar microstructural features. The volume is a cube with 25 53 voxels per side and $9^{\circ}$ corner-to-corner misorientation. The transition occurs linearly along the

54 middle third of the cube diagonal. The data has been made more realistic by contaminating 55 voxels with uniformly distributed noise to add, on average, $0.5^{\circ}$ misorientation from its original 56 orientation. This value was selected because, although angular accuracy of $0.01^{\circ}$ is possible with 57 high resolution methods, $0.5^{\circ}$ is typical for standard methods [5]. Segmentation by thresholding 58 is demonstrated in Figures $1 \mathrm{~b}$ and c. A threshold angle of $0.5^{\circ}$ finds many superfluous 59 boundaries, and a threshold angle of $0.75^{\circ}$ finds very few boundaries, all of which are from the 60 added noise. In both cases, noise dominates and no threshold value between improves the 61 situation. We present a robust solution to segmenting 3D EBSD data, which identifies the proper 62 boundary geometry such as that shown in Figure 1d. 
64 The challenges in characterizing fine-scale microstructures have been addressed by Humphreys

65 et al. [6] with a modified Kuwahara filter. This method passes through the data set calculating

66 the average and variance of every neighborhood (e.g. $3 \times 3$ or $3 \times 3 \times 3$ region). Then every point is

67 replaced with the average value of the neighborhood to which it belongs having the lowest

68 variance. This serves to reduce noise in the data set while preserving edges. Further processing,

69 including multiple passes of the filter, may be carried out before segmenting the data using a

70 misorientation threshold, as described above. While this may be sufficient in many cases, the

71 Kuwahara filter is local in nature, and the final thresholding step may produce too many edges in

72 one region of a data set and not enough in another. Furthermore, the results are unpredictable

73 over spatially gradual boundaries. The Kuwahara filter will be compared to our method below.

$75 \quad \mathbf{1 . 2} \quad$ Fast Multiscale Clustering

76 Fast multiscale clustering (FMC) is a segmentation technique developed for 2D image

77 processing [7]. The method creates a nested partitioning of the data set at many scales. Starting

78 with individual data points at the finest scale, the processes iteratively coarsens the partitioning

79 by combining groups. At each scale in the hierarchy, every group is then scored based on the

80 similarity of points it contains and distinction from neighboring groups. The highest-scoring

81 groups form the final segmentation of the data. FMC uses fuzzy logic in this grouping, so coarse

82 collections are not rigid. That is, a fine-scale group has probabilities of being part of more than

83 one coarse-scale group, and these probabilities are propagated through every scale. The

84 probability of assignment is based on the similarity of a group to certain neighboring groups that

85 are chosen as seeds for the coarser scale. Determining similarity of data requires a metric. For

86 simple color images, this can be a scalar difference between colors, but for application to EBSD 
87 data, the metric is based on misorientation. Here, we adapt FMC to work with a quaternion

88 representation for orientation data, so that misorientations can be calculated efficiently. A

89 detailed description of FMC and its modifications for EBSD data is provided in a 2D setting in

90 ref. [8], and the formulation extends to three dimensions. Although the iteration procedure is

91 probabilistic, selection of the final solution is deterministic, based on the optimal segmentation

92 of groups down to individual data points. Thus, the resulting reconstruction is reproducible over

93 repeated applications of the algorithm. The probabilities are also useful as a measurement of

94 confidence of each point's assignment in the final solution. Maps of this confidence can be

95 produced from the final segmentation data to reveal regions of high and low certainty.

97 At each scale in the hierarchy, groups inherit properties from the finer scale below, down to the

98 individual data points, all modified by the probability of assignment [9]. The aggregated

99 properties are the group size (volume), the average quaternion orientation, and the variance of

100 orientations within the group. This allows FMC to segment an entire data set with awareness of

101 local and global properties. Another advantage is that the method does not depend on the

102 physical spatial distribution of the data, only on the adjacency of points. Therefore, FMC is not

103 only applicable for 2D situations but easily applied in 3D. In fact, the data points do not have to

104 be located on a regular grid, as is the case after correcting for instrument drift, and neighbors can

105 be selected as any points within a given radius. Finally, since there are fewer groups at coarser

106 scales, FMC has a runtime linear with the number of data points. This is a practical consideration

107 that becomes important for methods applied to large data sets with more than two dimensions. 
109 A misorientation angle between average orientations is not useful on its own when comparing

110 coarse groups containing many data points. The Mahalanobis distance [10] is used to account

111 for additional information. This metric is defined as the pointwise distance between group

112 averages divided by the group variances. This gives groups with low variation naturally less

113 similarity to their neighbors than groups with high variance. Our modified definition for use with

114 EBSD data is the misorientation between the aggregated mean orientations divided by the

115 minimum of the group's variances. The minimum of the variances is used so that a group with a

116 high variance is not assigned a small distance to an entirely dissimilar group. A novel variance

117 aggregation method was developed by the authors [8] since the elements of quaternions are not

118 linearly correlated. While this value of Mahalanobis distance lacks physical significance, it can

119 be scaled at the aggregation step to give sense to closeness and farness, depending on the nature

120 of the data set. The scaling parameter, $\mathrm{C}_{\text {Maha }}$, determines the sensitivity of the segmentation [7].

121 Figure 2 shows segmentations of the artificially-constructed data set using different values of $122 \mathrm{C}_{\text {Maha }}$, along with confidence maps along the diagonal plane. In the more sensitive segmentation

123 with higher $\mathrm{C}_{\text {Maha }}$ (Figure $2 \mathrm{~b}$ ), the transition region is separate from the two simulated subgrain

124 regions that are uniform in orientation. In both cases, there is less confidence near the boundary

125 and the voxels there have nearly balanced probability of being assigned to either side. The band

126 of higher uncertainty is visibly wider in Figure 2a because the large cluster has larger variance in

127 orientation. This does not, however, necessarily indicate that the second segmentation is more

128 favorable, as one would also expect an inappropriate oversegmentation to have low variation

129 within the groupings. The process by which a $\mathrm{C}_{\text {Maha }}$ value of 2.5 (Figure $2 \mathrm{a}$ ) is chosen as optimal

130 is discussed below. 
132 FMC requires several other user-defined values, but fixed values for them have been effective

133 for all data sets examined in 2D and 3D. Hence, FMC has the additional advantage that it can be

134 controlled with a single parameter for application to different EBSD data sets, analogous to the

135 cutoff angle for a thresholding method. While high values of $\mathrm{C}_{\mathrm{Maha}}$ are effective for subgrain

136 structures, low values are optimal for segmentation of polycrystalline data along high-angle

137 boundaries. This has allowed validation of the method by performing it on data sets for which

138 thresholding is assumed to be effective. Validation in this way is shown in ref. [8] for a 2D slice

139 of $3 \mathrm{D}$ polycrystalline data. The entirety of this $3 \mathrm{D}$ data set has been subsequently segmented

140 with FMC, providing equally validating results. An additional 3D segmentation is presented in

141 Section 3.2 which identifies the visible grain boundaries in the data.

\section{2. Methods}

\section{$144 \quad \underline{2.1} \quad$ Preprocessing}

145 Some spatial preprocessing must be carried out since 3D EBSD data sets are collected in two 146 dimensional sections. Measurement skew and instrument drift are corrected with a bilinear 147 interpolation on each slice [11]. The data are then globally realigned, skewing the points off their 148 regular grid. Points then may be interpolated to a rectangular grid for computational efficiency, 149 but FMC can be performed with any spatial distribution of data points.

\section{$151 \quad \underline{2.2}$ Determining Optimal Sensitivity Parameter $\mathbf{C}_{\text {Maha }}$}

152 The sensitivity of the segmentation, controlled by $\mathrm{C}_{\text {Maha }}$, should be different based on the nature 153 of the data. Boundaries between clusters in polycrystalline data should be on the distinct grain 154 boundaries without consideration for variation within grains, but subgrain features are only 
155 captured with a more sensitive segmentation. There is no ground truth available for such a 156 segmentation, so the user is responsible for deciding which segmentation to use. Thus, an

157 automated method for determining the optimal value of $\mathrm{C}_{\text {Maha }}$ has been developed, inspired by 158 similar clustering problems in machine learning [12]. Oversegmentation is characterized by low 159 marginal variance change from splitting existing clusters. Therefore, an "elbow" in a plot of a 160 global variance measure as a function of $\mathrm{C}_{\text {Maha }}$ indicates a shift from creating clusters that should 161 be distinct towards erroneously breaking these clusters further. Once this elbow is identified, the 162 segmentation created using the associated value of $\mathrm{C}_{\text {Maha }}$ is selected as optimal.

163

164 The contribution of each cluster to the global variance metric is found by computing the variance 165 of misorientations for all points in the cluster from the cluster's mean orientation. Then these 166 variances are averaged over all clusters, weighted by the clusters' volumes, into a single global 167 variance metric [8].

\section{$169 \quad \underline{2.3}$ Surface Extraction}

170 When analyzing grain or subgrain volumes, it is often useful to isolate separate faces of an

171 irregular feature. These faces may be defined as regions having similar normal vectors, and, as

172 the FMC method developed operates on orientations, only a small further modification is needed 173 for application to normal vectors. With this modification, a second use of FMC identifies 174 coherent faces from the set of points on the exterior of a cluster.

175

176 The voxel locations from a 3D segment are used as the input for a volume reconstruction using 177 tentrahedra. The triangulated boundary surface is used to estimate local normal vectors, and 
178 these are used in the FMC process. As with the main use of FMC, the single $\mathrm{C}_{\text {Maha }}$ parameter

179 controls the sensitivity of the segmentation of the surface, and the same elbow criterion is used to

180 find the optimal segmentation that separates distinct faces without oversegmenting. It is pertinent

181 to note that it is a straightforward task to identify all of the large clusters in a volume and all of

182 the largest faces on those clusters, making study of many subgrain surfaces possible.

184 3. Results and Discussion

185 To demonstrate the utility of FMC for 3D EBSD data, we segment three data sets containing 186 significant microstructural features. In each set, the 2D EBSD scans are globally aligned with a 187 bilinear transformation [13] followed by nearest-neighbor interpolation to a rectangular grid with 188 the same spatial resolution as the original data. The interpolation is done to make the data 189 compatible with MTEX data structures [14], and is not a necessary step in general to perform 190 FMC. The optimal sensitivity parameter is then found and presented along with the resulting 191 segmentation. Finally, we select individual features from these segmentations to demonstrate the 192 FMC surface extraction method.

194 The FMC segmentations in this section were performed on a Tera multithreaded architecture 195 computer, and each took several hours. While the scalability of runtime is linear with the number 196 of data points, a 3D segmentation is slower than a 2D segmentation with the same number of 197 data points due to the increased connectivity among neighbors. This complexity, along with the 198 fact that an interesting 3D data set requires a large number of points, both contribute to lengthy 199 computations. Additionally, many independent segmentations must be performed if the optimal 200 sensitivity parameter is to be found for a given data set, but these may be done in parallel. 


\section{$202 \quad 3.1 \quad$ Microbands in Cold Rolled Aluminum}

203 We first apply FMC to segment microbands in a single grain of cold-rolled (22\% reduction)

204 commercial purity aluminum containing 1.1 million data points [13]. Figure 3 shows the EBSD

205 data with inverse pole figure coloring, in which the microbands are visible, as well as its

206 segmentation. The microband structure is captured by the FMC segmentation. The highlighted

207 microbands in Figure 3b are isolated in 3D in Figure 3c to show the nature of the interior of the 208 segmentation.

$210 \quad 3.2 \quad$ Recrystallized Grains in Cold Rolled and Partly Recrystallized Steel

211 Segmentation of a partly recrystallized extra low carbon (ELC) steel sample containing 350,000

212 data points [15] illustrates the ability of the FMC method to identify boundaries of different

213 magnitude in the same data set (Figure 4). Here, there are subtle subgrain features, distinct grain

214 boundaries, and a high angle boundary separating $\alpha$ - and $\gamma$-fiber regions. The presence of the

215 HAGB separating the regions does not affect the detection of boundaries with smaller

216 misorientation, even those adjacent to it. This is because variance comparisons are done between

217 each neighboring group, rather than with a global standard over the entire data set. The distinct 218 grain boundaries are identified as they would be with a misorientation thresholding 219 segmentation.

\section{$221 \quad 3.3 \quad$ Microbands in Deformed Nickel Single Crystal}

222 Microbands have been isolated in a 3D EBSD data set from a channel die plane strain 223 compressed (35\% strain) Goss-oriented nickel single crystal with more than 1.3 million data 
224 points [16]. The data have a standard deviation from the average orientation of only $0.7^{\circ}$. Figure

2255 shows the segmented microbands. Identification of these subgrain features would not be

226 attainable with thresholding at any angle. The segmentation in Figure $5 \mathrm{~b}$ is performed with the

227 optimal $\mathrm{C}_{\text {Maha. }}$. To show how even more subtle features may be identified, Figure 5c shows a

228 segmentation with a higher $\mathrm{C}_{\text {Maha }}$ value. This comes with the consequence of oversegmenting

229 the data overall, but specific features of interest may still be used for further analysis.

231 As an additional validation of the efficacy of the optimal segmentation, 2D sections have been

232 taken in the RD-ND plane, perpendicular to the original EBSD data. The spacing between

233 segment boundaries and the orientation of the bands agrees with TEM images of the microbands

234 within the sample [17]. Both of these observations are from global-scale spatial qualities of the

235 segmentation, which are not considered by the crystallographic orientation-based segmentation

236 method in any way.

237

238 This data set also has been segmented with the Kuwahara filter method [6]. The thresholding-

239 based process fails in application to 3D subgrain EBSD data because of the increased difficulty

240 of finding closed boundaries with the additional spatial dimension. What would be considered a

241 closed feature boundary in 2D is often resolved as an internal boundary in 3D. Segmentations of

242 the Kuwahara-filtered data are compared to FMC in Figures 6a and b, using the global variance

243 metric described in Section 2.2. The global variance decreases with increasing sensitivity in both

244 cases (higher $\mathrm{C}_{\mathrm{Maha}}$ or lower threshold), as expected (Figure 6). However, effective segmentation

245 methods with an adjustable parameter are expected to exhibit decreasing marginal changes in

246 variance, and the segmentations of the Kuwahara-filtered data do not. 
248 Further investigation into the Kuwahara-filtered data reveals that for the range of threshold

249 angles considered, a single, large cluster dominates the segmentation. This cluster occupies about

$25090 \%$ of the entire data set for all but the most dramatic oversegmentations. As the threshold

251 angle is decreased, the smaller clusters are split into progressively further oversegmented pieces

252 while the single large cluster continues to resist significant segmentation. The steep drop in

253 variance in Figure $6 \mathrm{~b}$ occurs with the separation of a crystallographically-distinct region from the

254 single dominating cluster. Although this region is large compared to the volume removed from

255 the large cluster in previous decrements in the threshold angle, it contains less than $2 \%$ of the

256 total remaining volume.

258 This behavior is further illustrated in Figures $6 \mathrm{c}$ and $6 \mathrm{~d}$. The two segmentation methods are 259 directly compared, using the total number of clusters produced in each application. With 260 progressively more sensitive FMC applications, regions of distinct orientation are isolated even 261 if point-to-point misorientation is low near their boundaries. On the other hand, the Kuwahara 262 filter method struggles to find closed boundaries satisfying the given threshold angle. Thus the 263 single large region remains unsegmented until the threshold is so low that useful subgrain

264 features are lost. Lastly, we note that applying the Kuwahara filter to a 3D data set is not 265 particularly faster than an FMC segmentation. However, the filter needs to be applied only once 266 before many thresholding segmentations can be carried out.

\section{$268 \quad \underline{3.4}$ Surface Segmentation}

269 The FMC surface extracting method was applied to microbands from the aluminum data set.

270 Figure 7 shows the top microband of Figure 3c, with the entire top and bottom surfaces isolated. 
271 These surfaces can be used for further geometric and crystallographic analysis. FMC is a 272 powerful tool for this application since it can form a full surface from similar components that 273 are separated by a sharp step in the estimated normal vectors.

274

\section{4. Concluding Summary}

276 The foregoing examples illustrate that fast multiscale clustering is an effective tool for 277 segmenting 3D EBSD data set containing subtle and gradual variations in orientation. The 278 Kuwahara filter, a common technique for segmenting this kind of data in 2D, does not generalize 279 well to three dimensions. To facilitate its use in the materials science community, we have 280 incorporated FMC modified to operate on orientation data into the free open source texture 281 analysis software package MTEX [14], thereby allowing users to take advantage of its existing 282 methods for importing and manipulating data. The algorithm is started by the command 283 calcGrains with argument FMC as grains = calcGrains (ebsd, 'FMC', C_Maha).

287 All EBSD segmentation described herein has been done with this package. The ability of the 288 method to segment data based on a wide range of boundary magnitudes with linear runtime make 289 FMC a robust method for both 2D and 3D segmentations. 


\section{Acknowledgements}

292 This work was supported by the Laspa Fellowship at Harvey Mudd College, the National 293 Science Foundation (DMR-0807240 and DGE-1144153), the Australian Research Council 294 Centre of Excellence for Design in Light Metals (CE05611574), the Harvey Mudd College 295 Department of Engineering and the Electron Microscope Unit at the University of New South 296 Wales. We acknowledge the preliminary FMC work of Cullen McMahon and Brian Soe. We 297 thank Benyue Liu, Donald Bolton and Nasima Afrin for contributions to collection of the 298 aluminum and nickel 3D EBSD data sets, Md. Zakaria Quadir and Nasima Afrin for discussion 299 of the nickel data set, and Wanqiang Xu for the steel data set.

301 References

302 [1] F. Bachmann, R. Hielscher, H. Schaeben, Grain detection from 2d and 3d EBSD dataSpecification of the MTEX algorithm, Ultramicrosc. 111 (2011) 1720-1733.

304 [2] S. Zaefferer, S. I. Wright. Three-dimensional orientation microscopy by serial sectioning and EBSD-based orientation mapping in a FIB-SEM, in: A.J. Schwartz, M. Kumar, B.L. Adams, D.P. Field (Eds.), Electron Backscatter Diffraction in Materials Science, second ed., Springer Science + Business Media LLC, New York 2009, pp. 109-122.

308 [3] A.D Rollett, S.B. Lee, R. Campman, G.S. Rohrer, Three-dimensional characterization of 309 microstructure by electron back-scatter diffraction, Annu. Rev. Mater. Res. 37 (2007) 627-

[4] M.A. Groeber, D.J. Rowenhorst, M.D. Uchic, Collection, processing, and analysis of threedimensional EBSD data sets, in: A.J. Schwartz, M. Kumar, B.L. Adams, D.P. Field (Eds.), 
315 [5] C. Maurice, R. Quey, R. Fortunier, J.H. Driver, High angular resolution EBSD and its 316 materials applications, in: D.A. Molodov (Ed.), Microstructural Design of Advanced 317 Engineering Materials, Wiley-VCH Verlag GmbH \& Co. KGaA, Weinheim, Germany, 2013, pp. 341-365.

[6] F.J. Humphreys, P. Bate, P. Hurley, Orientation averaging of electron backscattered diffraction data, J. Microsc. 201 (2001) 50-58

321 [7] D. Kushnir, M. Galun, A. Brandt, Fast multiscale clustering and manifold identification, Pattern Recognit. 39 (2006) 1876-1891.

323 [8] C. McMahon, B. Soe, A. Loeb, A. Vemulkar, M. Ferry, L. Bassman, Boundary 324 identification in EBSD data with a generalization of fast multiscale clustering, $325 \quad$ Ultramicrosc. 133 (2013) 16-25.

326 [9] E. Sharon, M. Galun, D. Sharon, R. Basri, A. Brandt, Hierarchy and adaptivity in segmenting visual scenes, Nature 442 (2006) 810-813.

[10] P.C. Mahalanobis, On the generalized distance in statistics,” Proc. Natl. Inst. Sci. India 2 (1936) 49-55.

[11] B. Soe, C. McMahon, D. Golay, M.Z. Quadir, M. Ferry, L. Bassman, Subgrain boundary identification in 3D EBSD data through fast multiscale clustering, Proc. First Int. Congr. 3D Mater. Sci. (2012) 189-194.

333 [12] C.A. Sugar, L.A. Lenert, R.A. Olshen, An application of cluster analysis to health services 334 research: empirically defined health states for depression from the sf-12, Technical 335 Report. Stanford Univ. (1999). 
[13] C. George, B. Soe, K. King, M.Z. Quadir, M. Ferry, L. Bassman, 3D microband boundary alignments and transitions in a cold rolled commercial purity aluminum alloy, Mater. Charact. 79 (2013) 15-21.

[14] F. Bachmann, R. Hielscher, H. Schaeben, Texture analysis with MTEX - free and open source software toolbox, Solid State Phenom. 160 (2010) 63-68.

[15] W. Xu, M.Z. Quadir, M. Ferry, A high-resolution three-dimensional electron backscatter diffraction study of the nucleation of recrystallization in cold-rolled extra-low-carbon steel, Metall. Mater. Trans. A 40A (2009) 1547-1556

[16] N. Afrin, M.Z. Quadir, L. Bassman, J.H. Driver, A. Albou, M. Ferry, The threedimensional nature of microbands in a channel die compressed Goss-oriented Ni single crystal, Scr. Mater. 64 (2011) 221-224.

[17] N. Afrin, M.Z. Quadir, M. Ferry, On the Complexities of Microband Interactions in a Cold-Deformed Goss-Oriented Ni Single Crystal, Metall. Mater. Trans. B 45 (2014) 345351. 


\section{Figure Captions}

353 Figure 1: (a) A constructed data set; (b) Segmentation with a threshold angle of $0.5^{\circ}$, finding 626 354 segments, and (c) $0.75^{\circ}$, finding 466 segments. The body has been made translucent and single355 voxel segments are removed to display the segments due to noise. (d) Segmentation using fast 356 multiscale clustering produces two clusters with a diagonal boundary.

358 Figure 2: The constructed data set from Figure 1, segmented with FMC using $\mathrm{C}_{\text {Maha }}$ values of (a) 3592.5 and (b) 5. The associated confidence maps are taken along the highlighted diagonal plane. 360 Darker pixels signify higher uncertainty.

362 Figure 3: 3D view of the cold rolled aluminum data set: (a) EBSD data; (b) EBSD data with 363 overlaid boundaries of segments (black and white lines) found with the optimal $\mathrm{C}_{\text {Maha }}$ value of 364 4.4, and (c) eight selected clusters, indicated with white lines in (b), showing the underlying 365 subgrain structure, including a boundary plane that extends through the entire data set. The 366 inverse pole figure color map shown is used for all figure colorings. (Scale is in microns).

368 Figure 4: 3D view of the partly recrystallized ELC steel data set: (a) EBSD data using the IPF 369 color map of Figure 3; (b) segmentation with the optimal $\mathrm{C}_{\text {Maha }}$ value of 3.4, and (c) boundaries 370 from the segmentation colored by local misorientation, showing the HAGB between $\alpha-$ and $\gamma-$ 371 fiber regions. (Scale is in microns). 
373 Figure 5: 3D view of the nickel data set (a) EBSD data, (b) segmentation with the optimal $\mathrm{C}_{\text {Maha }}$

374 value of 6 , (c) segmentation with a $C_{\text {Maha }}$ value of 7 , showing the identification of more subtle 375 features.

377 Figure 6: Effect of segmentation sensitivity on the global variance measure for 3D nickel data 378 under (a) FMC with $\mathrm{C}_{\text {Maha }}$ variation (optimal value shown with a solid dot) and (b) Kuwahara379 filtered segmentation with threshold angle variation. The threshold angle axis is reversed so that 380 segmentation sensitivity increases to the right. Direct comparison with the total number of 381 clusters using (c) the global variance measure, and (d) the size of the largest cluster produced by 382 each segmentation. Solid dots again indicate the optimal $\mathrm{C}_{\mathrm{Maha}}$ value. Note that the behavior 383 under a sequence of FMC segmentations is not necessarily monotone, but easily-identifiable 384 trends exist. Unlike thresholding, boundaries that exist at a coarser segmentations may change 385 under more sensitive segmentations.

387 Figure 7: Two views of a cluster in the segmentation of cold rolled aluminum data. Colors 388 indicate separate extraced surfaces. The top and bottom surfaces are isolated, as well as flat 389 surfaces on the top and side from the boundary faces of the data set. 
Figure 1

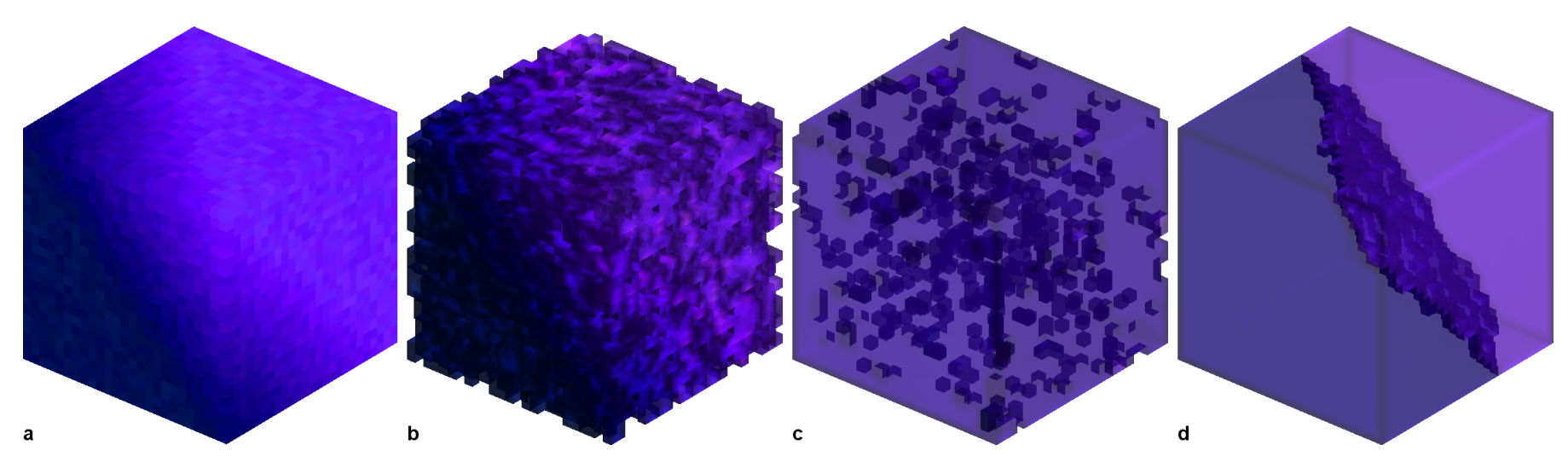


Figure 2

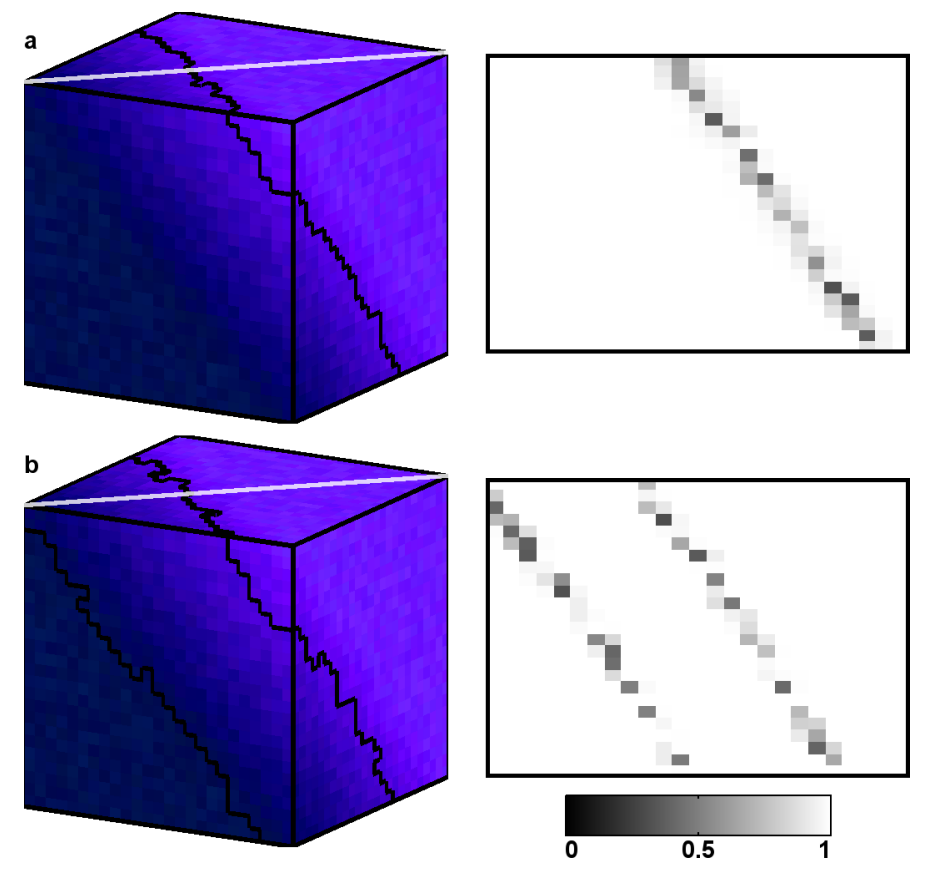


Figure 3

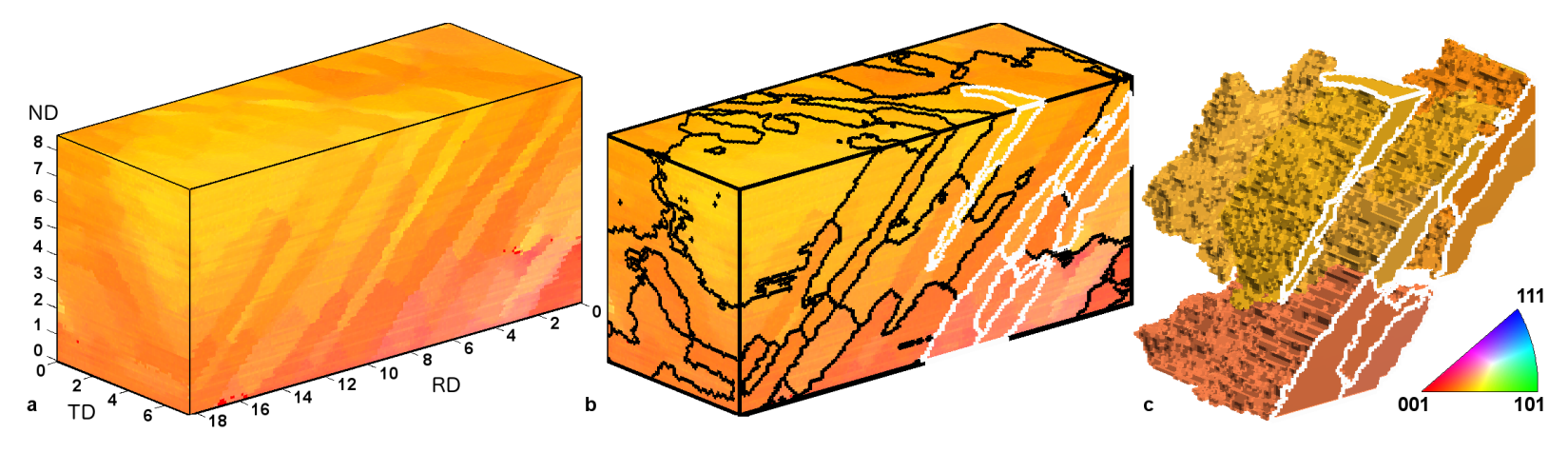


Figure 4
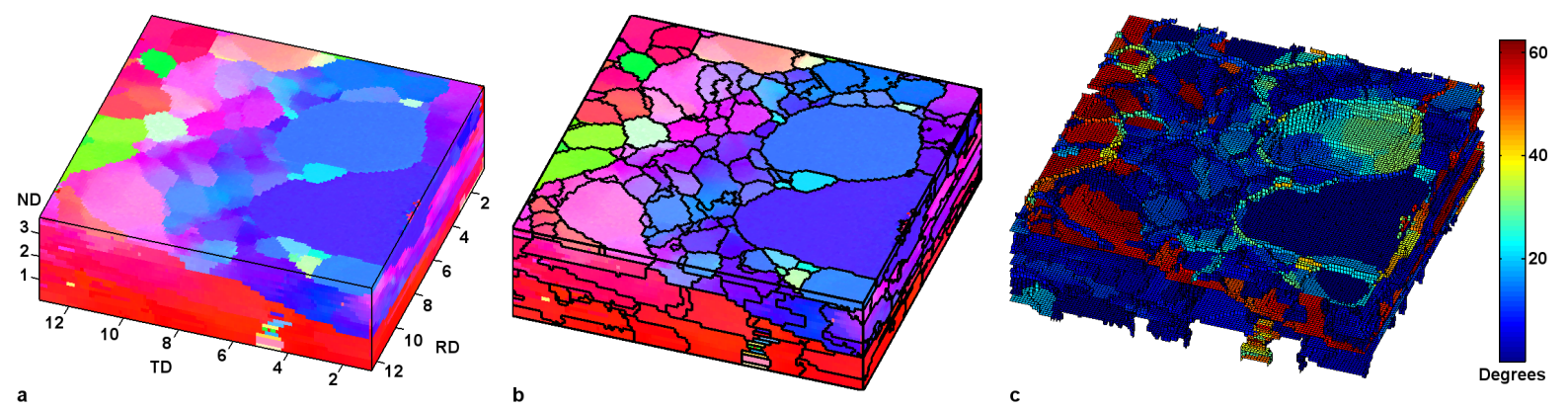
Figure 5

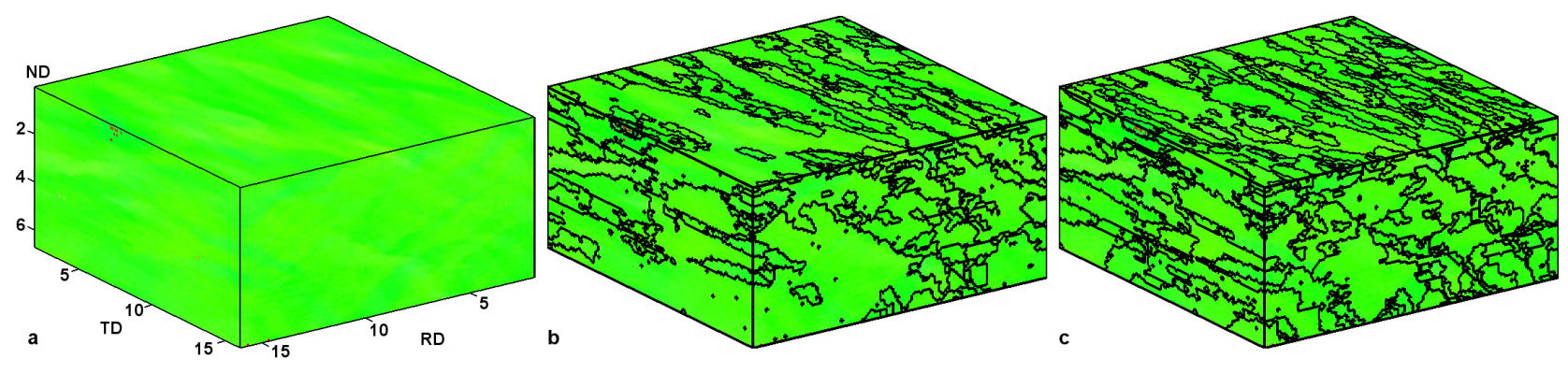


Figure 6
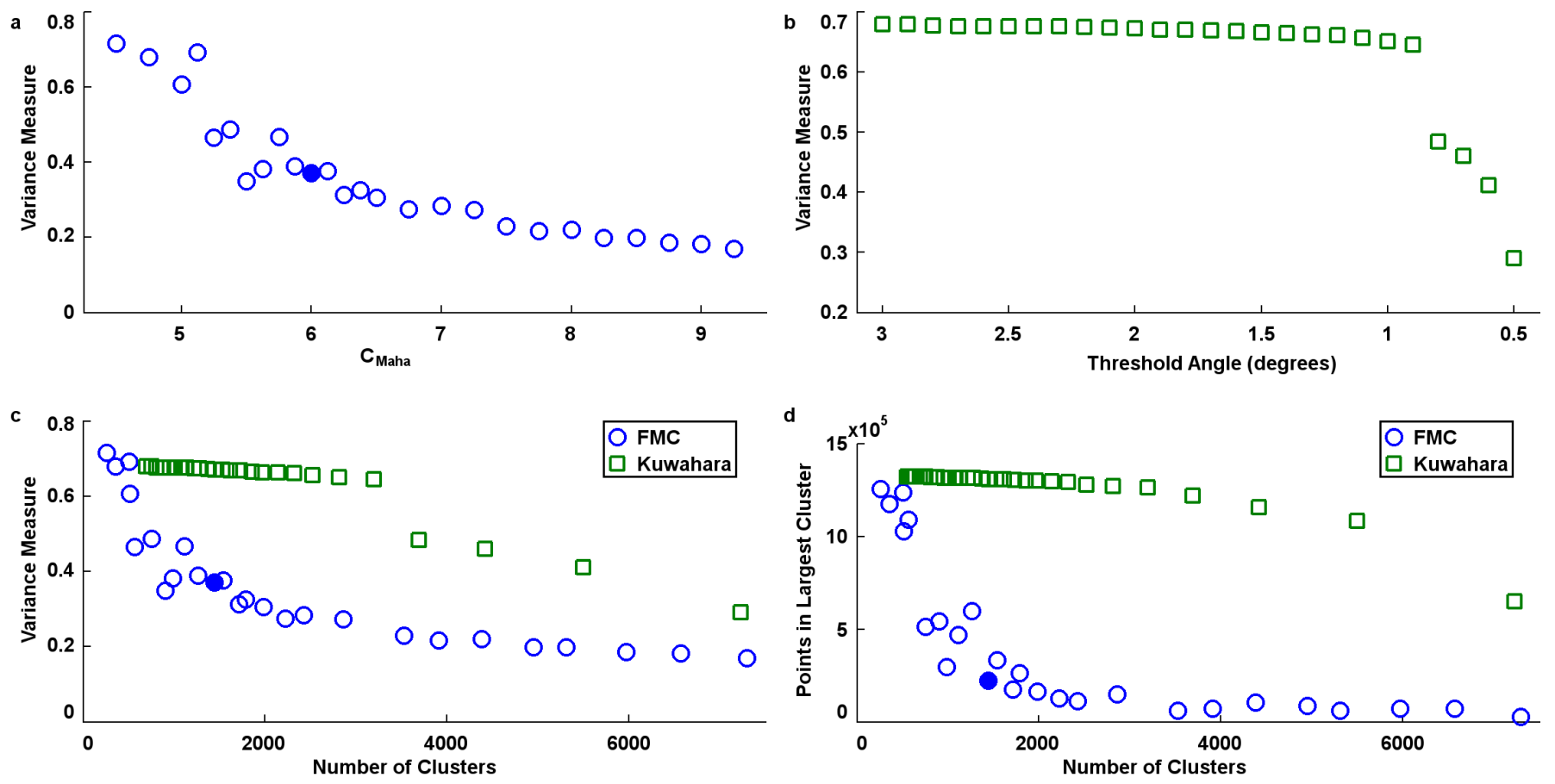
Figure 7

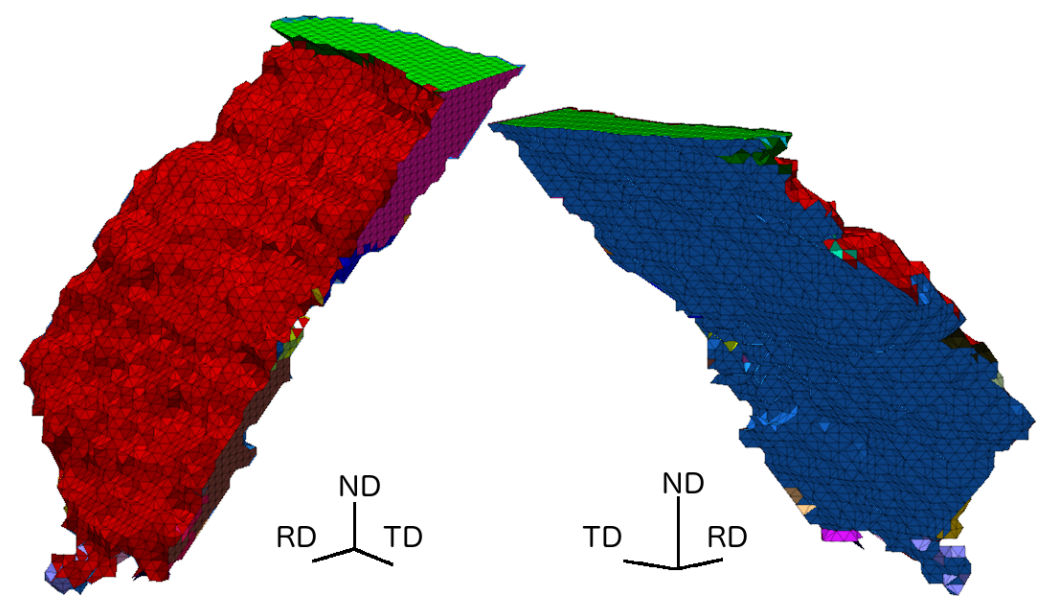

